\title{
Tom70 imports antiviral immunity to the mitochondria
}

\author{
Rongtuan Lin ${ }^{1,2}$, Suzanne Paz ${ }^{1,3}$, John Hiscott ${ }^{1,2,3}$ \\ ${ }^{I}$ Terry Fox Molecular Oncology Group, Lady Davis Institute for Medical Research; ${ }^{2}$ Department of Medicine; ${ }^{3}$ Department of \\ Microbiology \& Immunology, McGill University, Montreal, Canada \\ Cell Research (2010) 20:971-973. doi:10.1038/cr.2010.113; published online 13 July 2010
}

RLRs - the retinoic acid-inducible gene-I (RIG-I) and melanoma differentiation-associated gene 5 (MDA5) - are novel cytoplasmic RNA helicases that recognize viral RNA present within the cytoplasm [1]. The identification of the MAVS (mitochondrial antiviral signaling) adaptor protein as a member of the RIG-I-like receptors (RLRs) signaling pathway links the mitochondria to the mammalian antiviral defense system [2]. MAVS contains an amino-terminal CARD that interacts with the CARD domains of RIG-I/Mda5 and a carboxylterminal transmembrane domain that targets MAVS to the outer mitochondria. The localization of MAVS acts as a pivotal scaffold for triggering the antiviral cascade via activation of the transcription factors NF- $\kappa B$, IRF3 and IRF7 (Figure 1). Interestingly, MAVS is also required for nucleotide binding oligomerization domain 2 (NOD2)mediated ssRNA- or virus-induced activation of IFN production and antiviral response [3]. Recently, Dixit et $a l$. demonstrated that MAVS can also localize on peroxisomes [4]. In response to virus infection, peroxisomal MAVS rapidly induces expression of a subset of ISGs through an IFN-independent signaling pathway that provides shortterm protection until mitochondrial

Correspondence: Rongtuan Lin ${ }^{\mathrm{a}}$, John Hiscott ${ }^{\mathrm{b}}$

a्E-mail: rongtuan.lin@mcgill.ca

bE-mail: john.hiscott@mcgill.ca
MAVS induces a sustained antiviral response. At the mitochondria, MAVS can orchestrate the formation of a mitochondrial platform where multiple signaling molecules, such as TRAF2/3/5/6, TRADD, FADD, NEMO, RIP1, TANK and the recently identified MITA, that converge to mediate the activation of the classical IKK $\alpha / \beta$ and/or IKK-related kinases TBK1/IKKE (Figure 1) [5-6]. Two mitochondrial proteins, NOD-like receptor family member X1 (NLRX1) and $\mathrm{gClqR}$, have been recently identified as regulators of MAVS function and involved in inhibition of RIG-I and MDA5-dependent antiviral response [5]. Fascinatingly, the autophagy-related protein Atg5/12 and several other cellular proteins have also been implicated in the negative regulation of MAVS signaling (Figure 1). But exactly how MAVS is activated in this system and how it signals to downstream signaling complexes remains to be clarified. Understanding the composition and spatiotemporal events surrounding MAVS is essential to our comprehension of the IFN and antiviral response.

Using a proteomics approach, Liu and colleagues show in this issue of Cell Research that Tom70, a mitochondrial import receptor, interacts with MAVS and plays an important role in recruiting antiviral proteins involved in the innate immune response to virus infection [7]. The Tom (translocase of the outer membrane) complex is essential for the initial recognition of mitochondrial pre-proteins in the cytoplasm and is a "universal gate" for protein trafficking to the mitochondria [8]. Along with the many interacting partners described above, the study of Liu et al. argues that Tom70 can be added to the list of proteins involved in the regulation of MAVS-dependent antiviral signaling (Figure 1). Two mitochondrial preprotein import receptors - Tom 20 and Tom70 recognize their substrates via different mechanisms: Tom 20 generally interacts with pre-proteins containing a classical N-terminal signal peptide; whereas Tom70 recognizes internal signal sequences of pre-proteins that are often associated with the chaperone heat shock protein Hsp90 [9]. To determine whether other unknown adaptor proteins on the outer mitochondrial membrane are involved in MAVS-mediated signaling, Liu and colleagues immunoprecipitated mitochondrial transfected Flag-tagged MAVS and identified a $\sim 80$ $\mathrm{kDa}$ protein associated with the MAVS complex. This protein was subsequently identified as Tom 70 by mass spectrometry analysis. They also demonstrated that endogenous forms of Tom70 could interact with MAVS, and this association was significantly increased following virus infection. They determined the interaction to be mediated through the transmembrane domains of both MAVS and Tom70. In addition, the authors also detected the binding of Tom70 with TRADD, TRAF6, STING and IRF3 in an overexpression system 
RIG-I/MDA5/NOD2

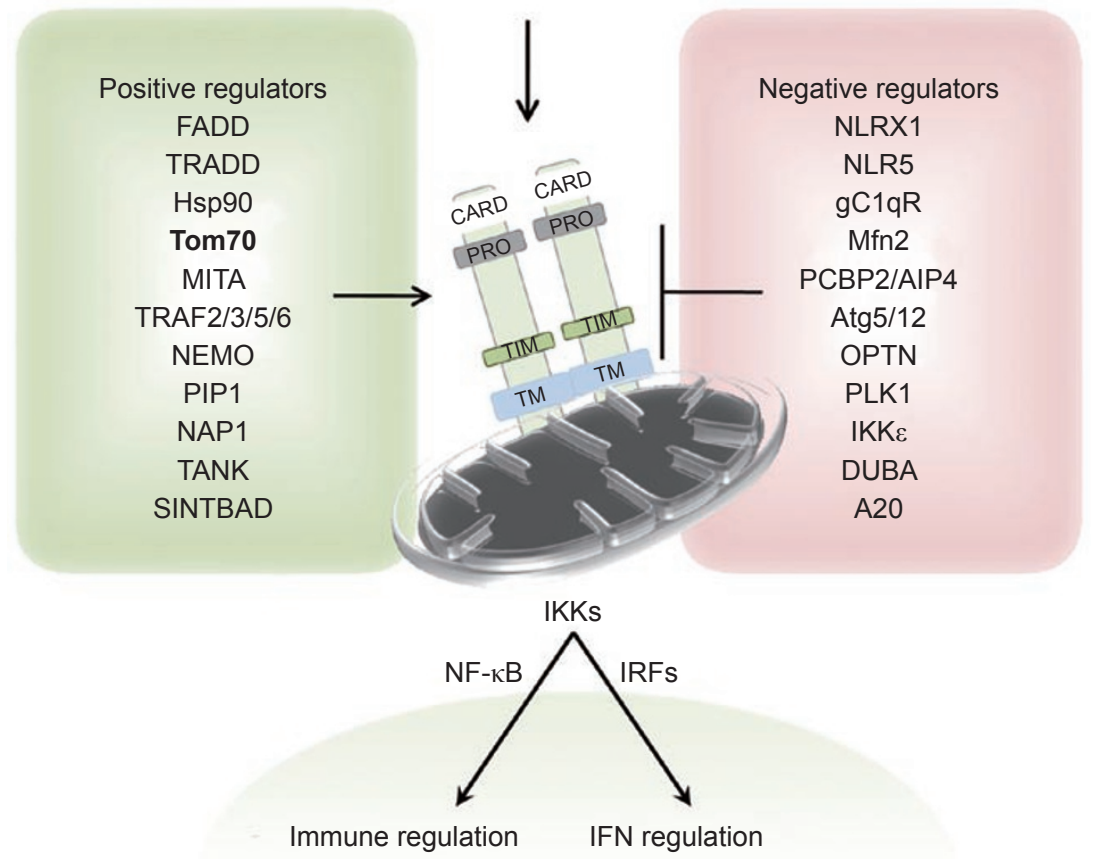

Figure 1 Regulation of MAVS signaling. RIG-I senses viral RNA through its helicase domain and relays downstream signals via its $\mathrm{N}$-terminal CARD domain to the mitochondrial adaptor MAVS. Many proteins have been implicated in the positive (green, left) or negative (red, right) regulation of MAVS signaling. MAVS triggers the activation of IKK-related kinases, TBK1/IKKE and the canonical IKK $\alpha / \mathrm{IKK} \beta$ complex. Activated TBK1 and IKKe phosphorylate IRF3 and IRF7, which provoke ISRE activation, IFN production, and adoption of an antiviral state. Activation of the canonical IKK $\alpha / \mathrm{IKK} \beta$ complex results in the release of $\mathrm{NF}-\mathrm{\kappa B}$ and activation of pro-inflammatory gene transcription.

using HEK293 cells.

To determine the involvement of Tom70 in MAVS-mediated signaling, Liu and colleagues undertook two approaches: 1) the effect of overexpressed Tom70 on RIG-I signaling; 2) the effect of knocking down Tom70 on Sendai Virus (SeV) infection. Overexpression of Tom70 enhanced IFNB, IFIT1 and RANTES gene expression induced by either $\mathrm{SeV}$ or poly (I:C) transfection in all HEK293, BMDM and BMDC cells. However, Tom70 expression had no influence on TRIF-mediated activation of IFN- $\beta$ promoter. Knockdown of Tom70 by RNA interference led to an inhibition of SeV-mediated IFNB, IFIT1 and RANTES gene expression profile; thus demonstrating a need for Tom70 in RIG-I-dependent signal- ing to the IFN and antiviral response. Consistent with the role of Tom70 in the regulation of interferon production, exogenous expression of Tom70 was able to block VSV and NDV replication, while knockdown of Tom70 expression enhanced virus replication. Together, these experiments support a role for Tom70 in RIG-I/MAVS-dependent antiviral signaling.

The IKK-related kinases - TBK1 and $\mathrm{IKK} \varepsilon$ - are critical downstream components of the activation of the interferon antiviral response, through their ability to phosphorylate the Cterminal domains of IRF3 and IRF7 (Figure 1). Previous studies from the Chen Wang's laboratory demonstrated that Hsp90 formed a dynamic complex with IRF3 and TBK1 essential for virus-mediated IRF3 activation [10]. Furthermore, another study by Young et al. demonstrated that through the chaperone binding dicarboxylate clamp domain in Tom70, Hsp90 in cooperation with Hsp70 mediated the targeting of a subset of mitochondrial pre-proteins to the Tom70 receptor [11]. These observations prompted the authors to examine the possibility that Tom70 could recruit TBK1 and/or IRF3 dynamically to mitochondrial platform via interaction with Hsp90. Using co-immunoprecipitation, Liu and colleagues revealed that Tom 70 associated with Hsp90, IRF3 and TBK1. Interestingly, knockdown of Hsp90 by siRNA dramatically decreased the association of Tom70 with TBK1 and IRF3. Mutational analysis revealed that the clamp domain of Tom70 and the EEVD motif of Hsp90 were essential domains mediating protein-protein interactions and virus-induced IRF3 activation. Moreover, in vitro GST pull-down confirmed that Hsp90 interacted directly with Tom70 or IRF3, and that Tom70 interacted with IRF3 only in the presence of Hsp90. The authors thus concluded that Tom70 recruits TBK1/IRF3 via Hsp90, both in vivo and in vitro.

Although this report convincingly demonstrates a role for Tom70 as a mitochondrial adaptor in virus-mediated activation of IFN responses, the underlying mechanism remains to be further clarified. Albeit Tom70 appears to be an important translocase protein linking MAVS with TBK1 and IRF3, the evidence does not demonstrate that TBK1 and/or IRF3 are recruited to either the mitochondria or MAVS. Because of its central role in protein import to the mitochondria, knockdown of Tom70 will affect the import of many mitochondria proteins, thus impacting on numerous cellular processes including apoptosis and energy metabolism. Silencing of Tom70 had no effect on mitochondrial localization of MAVS per se, but the distribution of MAVS appeared quite different when compared to wild-type cells (Figure 4F in reference [7]). One 
unresolved question is whether the abnormal distribution of MAVS (or other mitochondrial proteins) contributed to the inhibition of IRF3 activation? Another potential function of Tom70 in mitochondrial import of IRF3 is apoptosis as it was suggested by the recent study from the laboratory of Ganes Sen, who identified a BH3-like domain in IRF3 that enables activated IRF3 to associate with the pro-apoptotic BCL-2 family member Bax [12]. The Bax-IRF3 interaction triggered its translocation to the mitochondria and activation of the intrinsic mitochondrial apoptotic pathway. It would be interesting to determine whether Tom70 contributes to Bax-IRF3 recruitment to the mitochondria and apoptosis. Altogether, the discovery of the mitochondrial import protein Tom70 as a player in MAVSmediated signaling provides a new perspective on the regulation of antiviral immunity and further emphasizes the complexities associated with MAVSrelated mitochondrial interactions and the regulation of host immune response and apoptosis.

\section{References}

1 Yoneyama M, Kikuchi M, Natsukawa $\mathrm{T}$, et al. The RNA helicase RIG-I has an essential function in doublestranded RNA-induced innate antiviral responses. Nat Immunol 2004; 5:730737.

2 Seth RB, Sun L, Ea CK, Chen ZJ. Identification and characterization of MAVS, a mitochondrial antiviral signaling protein that activates NFkappaB and IRF 3. Cell 2005; 122:669682.

3 Sabbah A, Chang TH, Harnack R, et al. Activation of innate immune antiviral responses by Nod2. Nat Immunol 2009; 10:1073-1080.

4 Dixit E, Boulant S, Zhang Y, et al. Peroxisomes are signaling platforms for antiviral innate immunity. Cell 2010; 141:668-681.

5 Scott I. Mitochondrial factors in the regulation of innate immunity. Microbes Infect 2009; 11:729-736.

6 Tang ED, Wang CY. TRAF5 is a downstream target of MAVS in antiviral innate immune signaling. PLoS One
2010; 5:e9172.

7 Xin-Yi Liu, Bo Wei, He-Xin Shi, YuFei Shan, Chen Wang. Tom70 mediates activation of interferon regulatory factor 3 on mitochondria. Cell Res 2010; doi:10.1038/cr.2010.103.

8 Baker MJ, Frazier AE, Gulbis JM, Ryan MT. Mitochondrial proteinimport machinery: correlating structure with function. Trends Cell Biol 2007; 17:456-464.

9 Abe Y, Shodai T, Muto T, et al. Structural basis of presequence recognition by the mitochondrial protein import receptor Tom20. Cell 2000; 100:551-560.

10 Yang K, Shi H, Qi R, et al. Hsp90 regulates activation of interferon regulatory factor 3 and TBK-1 stabilization in Sendai virus-infected cells. Mol Biol Cell 2006; 17:1461-1471.

11 Young JC, Hoogenraad NJ, Hartl FU. Molecular chaperones Hsp90 and Hsp70 deliver preproteins to the mitochondrial import receptor Tom70. Cell 2003; 112: 41-50.

12 Chattopadhyay S, Marques JT, Yamashita $\mathrm{M}$, et al. Viral apoptosis is induced by IRF-3-mediated activation of Bax. EMBO J 2010; 29:1762-1773. 